\title{
Investigation of the basic catalytic activity of natural phosphates in the Michael condensation
}

\author{
Mohamed Zahouily, ${ }^{*}$ Bahija Mounir, Hind Charki, Abdessamad Mezdar, \\ Bouchaib Bahlaouan, and Mohamed Ouammou
}

Laboratoire de Catalyse,Chimiométrie et Environnement, UFR de Chimie Appliquée, Université Hassan II, Faculté des Sciences et Techniques, Mohammadia B. P. 146, 20650 Maroc

E-mail:mzahouily@yahoo.fr

\begin{abstract}
The effect of the specific surface area of natural phosphate on Michael condensations involving chalcone derivatives and various nucleophiles is reported. The catalytic activity is intimately connected to the specific surface area of the catalyst. The best activities were observed with the thermally treated micronised catalyst $\left(\mathrm{NP}_{\mathrm{m}}\right)$.
\end{abstract}

Keywords: Specific surface area, Michael condensation, natural microphosphate, heterogeneous catalysis

\section{Introduction}

The Michael condensation of a nucleophilic compound and an $\alpha, \beta$-unsaturated carbonyl compound is a convenient route for the construction of carbon-carbon and carbon-heteroatom bonds. ${ }^{1}$

In heterogeneous catalysis this reaction is carried under conditions which do not require separation of the product from other organic materials (such as the base), which are fast, effective and which do not require heating or the use of large quantities of solvent. Fluoroapatite, ${ }^{2}$ synthetic phosphate $\mathrm{Na}_{2} \mathrm{CaP}_{2} \mathrm{O}_{7},{ }^{3}$ aluminium oxide, ${ }^{4} \mathrm{Mg}$-Al hydrotalcite, ${ }^{5}$ and other catalysts have been tried with some success. ${ }^{6}$

More recently, we have shown that doping with mineral salts increases the activity of solid catalysts. ${ }^{7}$ We have shown also that the catalytic activity is intimately related to the structure of the solid catalysts. ${ }^{8}$ Other researchers have augmented the effect of the catalyst with the addition of ultrasound ${ }^{9}$ and microwave radiation. ${ }^{10}$

In the present work, our goal is to understand the influence of the specific surface area of the natural phosphate (NP) on the effective basicity of NP materials by comparison of their respective catalytic activity towards the Michael condensation between various nucleophiles 
(malononitrile, nitromethane, thiophenol, o-aminothiophenol, benzylamine and aniline) and chalcone derivatives (Scheme 1).

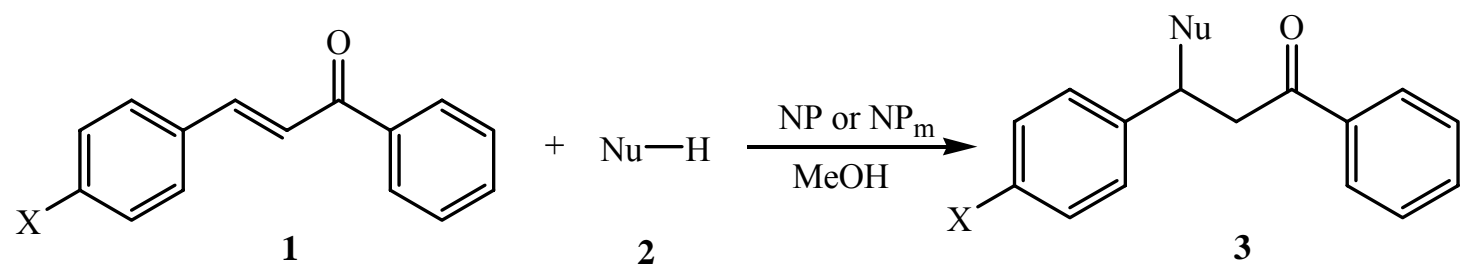

\section{Scheme 1}

\section{Results and Discussion}

We have carried out the Michael condensation in the presence of $\mathrm{NP}$ and $\mathrm{NP}_{\mathrm{m}}$. The results obtained in the condensation of Chalcone derivatives $\mathbf{1}$ with various nucleophiles $\mathbf{2}$ in the presence of $0.1 \mathrm{~g}$ of NP are shown in Table 1.

The NP shows an interesting catalytic activity and yields up to $60 \%$ are obtained in some cases (3a-e, 3g-h, 3j-1, 3n and 3p). Nevertheless, for the synthesis of compounds $\mathbf{3 f}$, 3i, 3m, $3 \mathbf{0}$ and 3q-t, the yields are moderate or low. In general, the reactions are relatively slow.

Except in one case, all the combinations lead selectively to the corresponding expected 1.4adducts. No by-products resulting from the undesirable 1.2-addition and/or bis-addition side reactions (usually observed under classical conditions in some cases) were observed. However, reaction of 2-aminothiophenol with a Michael acceptor bearing a nitro group ( 1 with $\mathrm{X}=\mathrm{NO}_{2}$ ) gives an $85 / 15$ mixture of 1.4- and 1.3-addition products (entry 14). The formation of this a priori unexpected regioisomer requires both a strong nucleophilic thiol and a strong electron-acceptor $\mathrm{X}$ group on the aromatic moiety of the chalcone derivative.

On the other hand, the increase of the specific surface area by the air jet micronic pulverisation process resulted in a yield improvement of $c a 20 \%$ compared to similar reactions catalysed with natural phosphates ${ }^{11}$ (Table 1). Moreover, the kinetic curves for the synthesis of the product $3 r$ in the presence of $\mathrm{NP}$ or $\mathrm{NP}_{\mathrm{m}}$ clearly show the enhancement of the catalytic activity of $\mathrm{NP}_{\mathrm{m}}$. The yields obtained after 6,10 and $16 \mathrm{~h}$ are 40,70, and $80 \%$ in the presence of $\mathrm{NP}_{\mathrm{m}}$ and 23,56 , and $58 \%$ in the presence of $\mathrm{NP}$. 
Table 1. Synthesis of products 3 by Michael condensation using NP and $\mathrm{NP}_{\mathrm{m}}$

\begin{tabular}{|c|c|c|c|c|c|}
\hline \multirow[b]{2}{*}{ Entry } & \multirow[b]{2}{*}{ Products } & \multirow[b]{2}{*}{$X$} & \multirow[b]{2}{*}{ Nucleophile } & \multicolumn{2}{|c|}{ Yield $/ \%(\text { time })^{\mathrm{a}}$} \\
\hline & & & & NP & $\mathrm{NP}_{\mathrm{m}}$ \\
\hline \multirow[t]{2}{*}{1} & $3 a$ & $\mathrm{H}$ & $(\mathrm{CN})_{2} \mathrm{CH}_{2}$ & $66(30 \mathrm{~min})$ & $74(30 \mathrm{~min})$ \\
\hline & & & & $84(45 \mathrm{~min})$ & $92(45 \mathrm{~min})$ \\
\hline \multirow[t]{2}{*}{2} & $\mathbf{3 b}$ & $\mathrm{Cl}$ & “ & $61(30 \mathrm{~min})$ & $70(30 \mathrm{~min})$ \\
\hline & & & & $77(45 \mathrm{~min})$ & $86(45 \mathrm{~min})$ \\
\hline 3 & $3 c$ & $\mathrm{OMe}$ & “" & $83(60 \mathrm{~min})$ & $90(60 \mathrm{~min})$ \\
\hline 4 & 3d & $\mathrm{H}$ & $\mathrm{NO}_{2} \mathrm{CH}_{3}$ & $73(6 h)$ & $84(6 h)$ \\
\hline 5 & $3 \mathbf{e}$ & $\mathrm{Cl}$ & “ & $62(7 h)$ & $70(7 \mathrm{~h})$ \\
\hline 6 & $3 f$ & OMe & “ & $48(7 h)$ & $60(7 h)$ \\
\hline 7 & $3 g$ & $\mathrm{H}$ & $\mathrm{PhSH}$ & $68(5 \min )$ & $96(5 \mathrm{~min})$ \\
\hline 8 & $3 h$ & $\mathrm{Cl}$ & “ & $74(5 \mathrm{~min})$ & $94(5 \mathrm{~min})$ \\
\hline 9 & $3 \mathbf{i}$ & $\mathrm{OMe}$ & “" & $50(60 \mathrm{~min})$ & $80(60 \mathrm{~min})$ \\
\hline 10 & $3 \mathbf{j}$ & $\mathrm{NO}_{2}$ & “" & $67(4 \min )$ & 97 (4min) \\
\hline 11 & $3 \mathbf{k}$ & $\mathrm{H}$ & $o-\mathrm{NH}_{2}-\mathrm{C}_{6} \mathrm{H}_{4} \mathrm{SH}$ & $92(1 \mathrm{~min})$ & $96(1 \mathrm{~min})$ \\
\hline 12 & 31 & $\mathrm{Cl}$ & “" & $90(1 \mathrm{~min})$ & $96(1 \mathrm{~min})$ \\
\hline 13 & $3 m$ & OMe & “ & $55(8 \min )$ & $78(8 \min )$ \\
\hline 14 & $3 n$ & $\mathrm{NO}_{2}$ & “ & $90(1 \mathrm{~min})^{\mathrm{b}}$ & $94(1 \min )^{b}$ \\
\hline 15 & 30 & $\mathrm{H}$ & $\mathrm{PhCH}_{2} \mathrm{NH}_{2}$ & $38(24 h)$ & $67(24 h)$ \\
\hline 16 & $3 p$ & $\mathrm{Cl}$ & “" & $66(24 h)$ & $72(24 h)$ \\
\hline 17 & $3 q$ & $\mathrm{OMe}$ & “ & $43(48 h)$ & $77(48 h)$ \\
\hline \multirow[t]{2}{*}{18} & $3 \mathbf{r}$ & $\mathrm{H}$ & $\mathrm{PhNH}_{2}$ & $23(6 h)$ & $40(6 h)$ \\
\hline & & & & $58(16 h)$ & $80(16 h)$ \\
\hline \multirow[t]{2}{*}{19} & 3s & $\mathrm{Cl}$ & “" & $20(6 h)$ & $42(6 h)$ \\
\hline & & & & $55(16 h)$ & $77(16 h)$ \\
\hline 20 & $3 t$ & $\mathrm{OMe}$ & “" & $57(20 \mathrm{~h})$ & $88(20 h)$ \\
\hline
\end{tabular}

${ }^{\mathrm{a}}$ Yields of pure products isolated by recrystallization with $\mathrm{AcOEt} / \mathrm{CH}_{2} \mathrm{Cl}_{2}$ or distillation under vacuum and identified by ${ }^{1} \mathrm{H},{ }^{13} \mathrm{C}$-NMR and IR spectroscopy. ${ }^{\mathrm{b}}$ The 1.4 -addition product/1.3addition product ratio is $85 / 15$. 
We compared the catalytic activity of NP and NPm in the Michael condensation of the chalcone derivatives 1 and various nucleophiles 2 in the presence of NP or NPm $(0.1 \mathrm{~g})$, respectively. For all cases, the best catalytic activity was observed with the natural microphosphate. This result shows that the catalytic activity is intimately related to the specific surface area of the catalyst.

Solid catalysts become particularly interesting when they can be regenerated. Indeed, in our case, $\mathrm{NP}_{\mathrm{m}}$ was recovered quantitatively by simple filtration and regenerated by calcination at $700^{\circ} \mathrm{C}$ during $15 \mathrm{~min}$. The recovered catalyst was reused several times without loss of activity, even after the seventh cycle product 3a was obtained with the same yield.

In summary, we believe that the micronisation of the natural phosphate represents a breakthrough in development of solid catalysts with regard to the environment. The catalytic activity of the phosphates tested appears largely related to the surface specific area of the catalyst to remove acidic or active hydrogen atom at the start of the mechanism.

\section{Experimental Section}

General Procedures. ${ }^{1} \mathrm{H}$ and ${ }^{13} \mathrm{C}$ NMR spectra were recorded at 400 and $100 \mathrm{MHz}$, respectively, on a Bruker DRX-400 spectrometer in $\mathrm{CDCl}_{3}$ as internal standard. The chemical shifts $(\delta)$ are expressed in ppm relative to $\mathrm{CDCl}_{3}$ and coupling constants $(J)$ in Hertz. Mass spectra were obtained on a TRACE DSQ (Thermo electron) mass spectrometer. IR spectra were obtained on a FTIR (ATI Mattson-Genesis Series) and reported in wave numbers $\left(\mathrm{cm}^{-1}\right)$. Surface area and pore size analysis were carried out at $77 \mathrm{~K}$ on a Micromeritics ASAP2010 instrument using nitrogen as adsorbent. X-ray diffraction patterns of the catalysts were obtained on a Philips 1710 diffractometer using CuKa radiation and SEM images were taken on a Hitachi S-2400 microscope. Melting points were determined with a "Thomas Hoover" melting (capillary method) apparatus and are uncorrected. Flash column chromatography was performed using Merck silica gel 60 (230-400 mesh ASTM).

All reactions were carried out under an atmosphere of air. Solvents and starting materials (Aldrich) were used without further purification.

\section{Preparation of the catalyst and structural characteristics}

We have chosen the Syrian natural phosphate that belongs to the family of apatitic phosphates with carbonated gangue belonging to the francolite group. The general formula is: $\mathrm{Ca}_{10-\mathrm{a}-}$ ${ }_{b} \mathrm{Na}_{\mathrm{a}} \mathrm{Mg}_{\mathrm{b}}\left(\mathrm{PO}_{4}\right)_{6-\mathrm{x}}\left(\mathrm{CO}_{3} \mathrm{~F}\right)_{\mathrm{x}} \mathrm{F}_{2}$ where: $\mathrm{a}$ and $\mathrm{b}$ : degrees of molar substitution of $\mathrm{Ca}$ by $\mathrm{Na}$ and $\mathrm{Mg}$ respectively and $\mathrm{x}$ : degree of substitution of $\mathrm{PO}_{4}$ by the combination $\left(\mathrm{CO}_{3}+\mathrm{F}\right)$. Apatite phosphate with carbonated gangue contains phosphate grains in the form of nodules and bone matter. $^{12}$

Natural microphosphate $\left(\mathrm{NP}_{\mathrm{m}}\right)$ was obtained by an air jet micronic pulverisation process: micronic pulverisation is an engineering process unit operation, which produces fine powders 
$\left(\mathrm{d}_{50}: 25\right.$ to $\left.0.1 \mu \mathrm{m}\right)$ at the same time as optimizing the reactive surface of the material by the creation of new surface. This is done by removing the old surface or by modifying the physicochemical nature of the surface (specific area and particle size). ${ }^{12,13}$

The structures of the NP and NPm were confirmed by X-ray diffraction and thermal analysis.

The BET specific surface area was found to be $S=15.2 \mathrm{~m}^{2} / \mathrm{g}$ and $28.7 \mathrm{~m}^{2} / \mathrm{g}$ before calcinations for NP and NPm, respectively.

\section{Typical experimental procedure}

To a flask containing an equimolar mixture $(1 \mathrm{mmol})$ of nucleophile $\mathbf{2}$ and chalcone derivative $\mathbf{1}$ in methanol $(1 \mathrm{~mL})$ was added $\mathrm{NP}$ or $\mathrm{NP}_{\mathrm{m}}(0.1 \mathrm{~g})$ and the mixture was stirred at room temperature until completion of the reaction, as monitored by thin layer chromatography (TLC). The reaction mixture was filtered and the catalyst washed with dichloromethane. After concentration of the filtrate under reduced pressure the residue was subjected to recrystallization $\left(\mathrm{AcOEt} / \mathrm{CH}_{2} \mathrm{Cl}_{2}\right)$ or distillation under vacuum leading to the Michael adduct. The product structure was analysed by ${ }^{1} \mathrm{H},{ }^{13} \mathrm{C}-\mathrm{NMR}$ and IR spectrometry.

\section{Identifications of Products}

2-(3-Oxo-1,3-diphenyl-propyl)malononitrile (3a) ${ }^{\mathbf{1 4}}$. White solid; mp 124-127 ${ }^{\circ} \mathrm{C}$; $\mathrm{Rf}(20 \%$ AcOEt/hexane) $0.31 ; v_{\max }(\mathrm{KBr}) 1596 ; 1696 ; 2263 \mathrm{~cm}^{-1} ; \delta_{\mathrm{H}}\left(400 \mathrm{MHz} \mathrm{CDCl}_{3}\right) 3.6\left(\mathrm{H}, \mathrm{CH}_{2}\right.$, dd. $\left.J_{1}=5.2 \mathrm{~Hz}, J_{2}=18.4 \mathrm{~Hz}\right) ; 3.7\left(\mathrm{H}, \mathrm{CH}_{2}, \mathrm{dd}, J_{1}=8.4 \mathrm{~Hz}, J_{2}=18.4 \mathrm{~Hz}\right) ; 3.9\left(\mathrm{H}, \mathrm{CH}, \mathrm{dt}, J_{1}=5.2\right.$ $\left.\mathrm{Hz}, J_{2}=8.4 \mathrm{~Hz}\right) ; 4.6(\mathrm{H}, \mathrm{CH}, \mathrm{d}, J=5.1 \mathrm{~Hz}) ; 7.2-7.4(5 \mathrm{H}$, arom, m) ; $7.5(2 \mathrm{H}$, arom, t, $J=7.6$ $\mathrm{Hz}) ; 7.6(\mathrm{H}$, arom, t, $J=7.6 \mathrm{~Hz}) ; 7.9(2 \mathrm{H}$, arom, d, $J=7.2 \mathrm{~Hz}) ; \delta_{\mathrm{C}}\left(100 \mathrm{MHz}_{\mathrm{CDCl}}\right) 28.7$; $40.0 ; 41.1 ; 111.6 ; 111.8 ; 127.9 ; 128.0 ; 128.9 ; 129.1 ; 129.3 ; 134.1 ; 136.4 ; 197.6 . \mathrm{MS}(\mathrm{m} / \mathrm{z})$ $154\left(\mathrm{M}^{+}-\mathrm{CH}_{3} \mathrm{COPh}\right) ; 127 ; 120\left(\mathrm{M}^{+}-\mathrm{C}_{6} \mathrm{H}_{5} \mathrm{CH}=\mathrm{C}(\mathrm{CN})_{2}\right) ; 105 ; 103 ; 77 ; 51 ; 44$.

2-[1-(4-Chlorophenyl)-3-oxo-3-phenylpropyl]malononitrile (3b). White solid, mp 115-117 ${ }^{\circ} \mathrm{C} ; \mathrm{R}_{\mathrm{f}}\left(20 \%\right.$ AcOEt/hexane) $0.25 ; v_{\max }(\mathrm{KBr}) 1605 ; 1687 ; 2263 \mathrm{~cm}^{-1} ; \delta_{\mathrm{H}}\left(400 \mathrm{MHz} \mathrm{CDCl}_{3}\right) 3.5$ $\left(\mathrm{H}, \mathrm{CH}_{2}, \mathrm{dd}, J_{1}=5.3 \mathrm{~Hz}, J_{2}=18.4 \mathrm{~Hz}\right) ; 3.6\left(\mathrm{H}, \mathrm{CH}_{2}, \mathrm{dd}, J_{1}=8.5 \mathrm{~Hz}, J_{2}=18.4 \mathrm{~Hz}\right) ; 3.9(\mathrm{H}, \mathrm{CH}$, $\left.\mathrm{dt}, J_{1}=5.2 \mathrm{~Hz}, J_{2}=8.4 \mathrm{~Hz}\right) ; 4.6(\mathrm{H}, \mathrm{CH}, \mathrm{d}, J=5.1 \mathrm{~Hz}) ; 7.3-7.4(4 \mathrm{H}$, arom, m) ; $7.5(2 \mathrm{H}$, arom, t, $J=7.3 \mathrm{~Hz}) ; 7.6(\mathrm{H}$, arom, t, $J=7.3 \mathrm{~Hz}) ; 7.9(2 \mathrm{H}$, arom, d, $J=8.5 \mathrm{~Hz}) ; \delta_{\mathrm{C}}(100 \mathrm{MHz}$ $\left.\mathrm{CDCl}_{3}\right) 29.2 ; 29.6 ; 39.9 ; 40.6 ; 111.4 ; 111.6 ; 128.0 ; 128.9 ; 129.3 ; 129.4 ; 130.0 ; 134.1$; $134.9 ; 135.1 ; 135.6 ; 196.2 ; \mathrm{MS}(\mathrm{m} / \mathrm{z}): 188\left(\mathrm{M}^{+}-\mathrm{CH}_{3} \mathrm{COPh}\right) ; 161 ; 153 ; 137 ; 126 ; 120\left(\mathrm{M}^{+}\right.$$\left.p-\mathrm{ClC}_{6} \mathrm{H}_{4} \mathrm{CH}=\mathrm{C}(\mathrm{CN})_{2}\right) ; 105 ; 99 ; 77 ; 51$.

2-[1-(4-Methoxyphenyl)-3-oxo-3-phenylpropyl]malononitrile (3c). White solid, mp 118-119 ${ }^{\circ} \mathrm{C} . \mathrm{R}_{\mathrm{f}}\left(20 \%\right.$ AcOEt/hexane) $0.20 ; \mathrm{v}_{\max }(\mathrm{KBr}) 1614 ; 1687 ; 2263 \mathrm{~cm}^{-1} ; \delta_{\mathrm{H}}\left(400 \mathrm{MHz} \mathrm{CDCl}_{3}\right) 3.6$ $\left(\mathrm{H}, \mathrm{CH}_{2}, \mathrm{dd}, J_{1}=5.8 \mathrm{~Hz}, J_{2}=18.6 \mathrm{~Hz}\right) ; 3.7\left(\mathrm{H}, \mathrm{CH}_{2}, \mathrm{dd}, J_{1}=8.3 \mathrm{~Hz}, J_{2}=18.6 \mathrm{~Hz}\right) ; 3.8(3 \mathrm{H}$, $\left.\mathrm{OCH}_{3}, \mathrm{~s}\right) ; 3.9\left(1 \mathrm{H}, \mathrm{CH}, \mathrm{dt}, J_{1}=5.2 \mathrm{~Hz}, J_{2}=8.4 \mathrm{~Hz}\right) ; 4.6(1 \mathrm{H}, \mathrm{CH}, \mathrm{d}, J=5.1 \mathrm{~Hz}) ; 7.0(2 \mathrm{H}$, arom, d, $J=7.5 \mathrm{~Hz}) ; 7.4(2 \mathrm{H}$, arom, d, $J=7.5 \mathrm{~Hz}) ; 7.5(2 \mathrm{H}$, arom, t, $J=7.1 \mathrm{~Hz}) ; 7,6(\mathrm{H}$, arom, $\mathrm{t}, J=7.3 \mathrm{~Hz}) ; 8.0(2 \mathrm{H}$, arom, d, $J=7.1 \mathrm{~Hz}) ; \delta_{\mathrm{C}}\left(100 \mathrm{MHz} \mathrm{CDCl}_{3}\right) 22.9 ; 25.6 ; 41.5 ; 56.0$; $113.8 ; 117.7 ; 127.3 ; 128.4 ; 128.6 ; 132.9 ; 137.4 ; 141.0 ; 159.2 ; 197.6 ; \mathrm{MS}(\mathrm{m} / \mathrm{z}): 184\left(\mathrm{M}^{+}-\right.$ $\left.\mathrm{CH}_{3} \mathrm{COPh}\right) ; 169 ; 141 ; 114 ; 120\left(\mathrm{M}^{+}-p-\mathrm{MeO}-\mathrm{C}_{6} \mathrm{H}_{4} \mathrm{CH}=\mathrm{C}(\mathrm{CN})_{2}\right) ; 77 ; 51$.

4-Nitro-1,3-diphenylbutan-1-one (3d) ${ }^{15}$. White solid, mp $57-59{ }^{\circ} \mathrm{C} ; \mathrm{R}_{\mathrm{f}}(20 \% \mathrm{AcOEt} / \mathrm{hexane})$ $0.6 ; v_{\max }(\mathrm{KBr}) 1495 ; 1550 ; 1605 ; 1660 ; 1687 \mathrm{~cm}^{-1} ; \delta_{\mathrm{H}}\left(400 \mathrm{MHz} \mathrm{CDCl}_{3}\right) 3,3\left(\mathrm{H}, \mathrm{CH}_{2}, \mathrm{dd}, J_{1}\right.$ 
$\left.=7.2 \mathrm{~Hz}, J_{2}=17.6 \mathrm{~Hz}\right) ; 3,4\left(\mathrm{H}, \mathrm{CH}_{2}, \mathrm{dd}, J_{1}=6.4 \mathrm{~Hz}, \mathrm{~J}_{2}=17.6 \mathrm{~Hz}\right) ; 4,2(\mathrm{H}, \mathrm{CH}, \mathrm{m}) ; 4,6(\mathrm{H}$, $\left.\mathrm{CH}, \mathrm{dd}, J_{1}=8 \mathrm{~Hz}, J_{2}=12.4 \mathrm{~Hz}\right) ; 4.7\left(\mathrm{H}, \mathrm{CH}, \mathrm{dd}, J_{1}=6.4 \mathrm{~Hz}, J_{2}=12.4 \mathrm{~Hz}\right) ; 7.2-7.3(5 \mathrm{H}$, arom, m) ; $7.4(2 \mathrm{H}$, arom, t, $J=7.2 \mathrm{~Hz}) ; 7,5(\mathrm{H}$, arom, t, $J=7.2 \mathrm{~Hz}) ; 7,9(2 \mathrm{H}$, arom, d, $J=7.2 \mathrm{~Hz}) ; \delta_{\mathrm{C}}$ $\left(100 \mathrm{MHz} \mathrm{CDCl}_{3}\right) 29.6 ; 39.2 ; 41.4 ; 79.5 ; 127.4 ; 127.8 ; 127.9 ; 128.3 ; 128.4 ; 128.6 ; 128.7$; $128.8 ; 129.0 ; 133.5 ; 136.3 ; 139.0 ; 196.7 ; \mathrm{MS}(\mathrm{m} / \mathrm{z}): 222\left(\mathrm{M}^{+}-\mathrm{NO}_{2}\right) ; 117 ; 105 ; 77 ; 51$.

3-(4-Chlorophenyl)-4-nitro-1-phenylbutan-1-one (3e) ${ }^{\mathbf{1 6}}$. White solid, mp 110-112 ${ }^{\circ} \mathrm{C} ; \mathrm{R}_{\mathrm{f}}(20 \%$ AcOEt/hexane) $0.56 ; v_{\max }(\mathrm{KBr})_{-} 1495 ; 1550 ; 1596 ; 1660 ; 1687 \mathrm{~cm}^{-1} ; \delta_{\mathrm{H}}\left(400 \mathrm{MHz} \mathrm{CDCl}_{3}\right)$ $3.3\left(\mathrm{H}, \mathrm{CH}_{2}, \mathrm{dd}, J_{1}=7.3 \mathrm{~Hz}, J_{2}=17.6 \mathrm{~Hz}\right) ; 3.4\left(\mathrm{H}, \mathrm{CH}_{2}, \mathrm{dd}, J_{1}=6.3 \mathrm{~Hz}, J_{2}=17.6 \mathrm{~Hz}\right) ; 4.2(\mathrm{H}$, $\mathrm{CH}, \mathrm{m}) ; 4.6\left(\mathrm{H}, \mathrm{CH}, \mathrm{dd}, J_{1}=8.1 \mathrm{~Hz}, J_{2}=12.6 \mathrm{~Hz}\right) ; 4.8\left(\mathrm{H}, \mathrm{CH}, \mathrm{dd}, J_{1}=6.8 \mathrm{~Hz}, J_{2}=12.6 \mathrm{~Hz}\right)$; 7.1-7.3 (4H, arom, m); $7.4(2 \mathrm{H}$, arom, t, $J=7.3 \mathrm{~Hz}) ; 7.5(\mathrm{H}$, arom, t, $J=7.3 \mathrm{~Hz}) ; 7.9(2 \mathrm{H}$, arom, d, $J=8.5 \mathrm{~Hz}) ; \delta_{\mathrm{C}}\left(100 \mathrm{MHz}_{\mathrm{CDCl}}\right) 28.7 ; 43.5 ; 81.9 ; 127.7 ; 128.4 ; 128.6 ; 131.0$; $132.9 ; 137.4 ; 146.8 ; 197.6$; $\mathrm{MS}(\mathrm{m} / \mathrm{z}): 257\left(\mathrm{M}^{+}-\mathrm{NO}_{2}\right) ; 207 ; 105 ; 77 ; 51$

3-(4-Methoxyphenyl)-4-nitro-1-phenylbutan-1-one (3f) ${ }^{\mathbf{1 7}}$. White solid; mp $61-63{ }^{\circ} \mathrm{C}$; $\mathrm{Rf}(20 \%$ AcOEt/hexane $\quad 0.25 ; \quad v_{\max } \quad(\mathrm{KBr}) \quad 1495 ; \quad 1550 ; 1614$; $1660 \mathrm{~cm}^{-1} ; 1687 ; \delta_{\mathrm{H}}\left(400 \mathrm{MHz} \mathrm{CDCl}_{3}\right) 3.3\left(\mathrm{H}, \mathrm{CH}_{2}, \mathrm{dd}, J_{1}=7.3 \mathrm{~Hz}, J_{2}=17.4 \mathrm{~Hz}\right) ; 3.4\left(\mathrm{H}, \mathrm{CH}_{2}\right.$, $\left.\mathrm{dd}, J_{1}=5.3 \mathrm{~Hz}, J_{2}=17.4 \mathrm{~Hz}\right) ; 3.8\left(3 \mathrm{H}, \mathrm{OCH}_{3}, \mathrm{~s}\right) ; 4.1(\mathrm{H}, \mathrm{CH}, \mathrm{m}) ; 4.6\left(\mathrm{H}, \mathrm{CH}, \mathrm{dd}, J_{1}=8.1 \mathrm{~Hz}\right.$, $\left.J_{2}=12.3 \mathrm{~Hz}\right) ; 4.7\left(\mathrm{H}, \mathrm{CH}, \mathrm{dd}, J_{1}=6.5 \mathrm{~Hz}, J_{2}=12.3 \mathrm{~Hz}\right) ; 7.0(2 \mathrm{H}$, arom, d, $J=7.5 \mathrm{~Hz}) ; 7.4$ $(2 \mathrm{H}$, arom, d, $J=7.5 \mathrm{~Hz}) ; 7.5(2 \mathrm{H}$, arom, t, $J=7.1 \mathrm{~Hz}) ; 7.6(\mathrm{H}$, arom, t, $J=7.3 \mathrm{~Hz}) ; 8.0(2 \mathrm{H}$, arom, d, $J=7.1 \mathrm{~Hz}) ; \delta_{\mathrm{C}}\left(100 \mathrm{MHz} \mathrm{CDCl}_{3}\right) 28.7 ; 43.5 ; 56.0 ; 81.9 ; 113.8 ; 127.3 ; 128.4$; $128.6 ; 132.9 ; 137.4 ; 141.0 ; 159.2 ; 197.6$; $\mathrm{MS}(\mathrm{m} / \mathrm{z}): 252\left(\mathrm{M}^{+}-\mathrm{NO}_{2}\right) ; 239 ; 224 ; 105 ; 77 ; 51$.

1,3-Diphenyl-3-phenylsulfenylpropan-1-one (3g). White solid, mp $116-118{ }^{\circ} \mathrm{C}$; $\mathrm{R}_{\mathrm{f}}(10 \%$ AcOEt/hexane) 0.36; $v_{\max }(\mathrm{KBr}) 1680 \mathrm{~cm}^{-1} ; \delta_{\mathrm{H}}\left(400 \mathrm{MHz}_{\mathrm{CDCl}}\right) 3.5\left(\mathrm{H}, \mathrm{CH}_{2}, \mathrm{dd}, J_{1}=6.2 \mathrm{~Hz}\right.$, $\left.J_{2}=17.2 \mathrm{~Hz}\right) ; 3.7\left(\mathrm{H}, \mathrm{CH}_{2}, \mathrm{dd}, J_{1}=8.1 \mathrm{~Hz}, J_{2}=17.2 \mathrm{~Hz}\right) ; 4.98(\mathrm{H}, \mathrm{CH}, \mathrm{t}, J=7.2 \mathrm{~Hz}) ; 7.2-7.6$ $\left(13 \mathrm{H}\right.$, arom, m) ; $7.9(2 \mathrm{H}$, arom, d, $J=7.5) ; \delta_{\mathrm{C}}\left(100 \mathrm{MHz} \mathrm{CDCl}_{3}\right) 44.7 ; 48.3 ; 127.4 ; 127.6$; $127.9 ; 128.1 ; 128.5 ; 128.6 ; 128.9 ; 132.8 ; 133.3 ; 134.6 ; 136.8 ; 141.3 ; 197.9 ; \mathrm{MS}(\mathrm{m} / \mathrm{z}): 318$ $\left(\mathrm{M}^{+}\right) ; 206 ; 109$.

3-(4-Chlorophenyl)-1-phenyl-3-phenylsulfenylpropan-1-one (3h). White solid; mp 64-67 ${ }^{\circ} \mathrm{C}$; $\mathrm{R}_{\mathrm{f}}\left(10 \%\right.$ AcOEt/hexane) $0.35 ; v_{\max }(\mathrm{KBr}) 1730 \mathrm{~cm}^{-1} ; \delta_{\mathrm{H}}\left(400 \mathrm{MHz} \mathrm{CDCl}_{3}\right) 3.6\left(2 \mathrm{H}, \mathrm{CH}_{2}, \mathrm{~m}\right)$; $4.97(\mathrm{H}, \mathrm{CH}, \mathrm{t}, J=7.2 \mathrm{~Hz}) ; 7.2-7.5\left(12 \mathrm{H}\right.$, arom, m) ; $7.9(2 \mathrm{H}$, arom, d, $J=7.5 \mathrm{~Hz}) ; \delta_{\mathrm{C}}(100$ $\left.\mathrm{MHz} \mathrm{CDCl}_{3}\right) 44.2 ; 47.8 ; 122.3 ; 122.7 ; 128.1 ; 128.3 ; 128.8 ; 129.1 ; 129.3 ; 132.9 ; 133.4$; $133.6 ; 134.2 ; 136.3 ; 143.8 ; 148.2 ; 196.1 ; \mathrm{MS}(\mathrm{m} / \mathrm{z}): 353\left(\mathrm{M}^{+}\right) ; 241.91 ; 109$.

3-(4-Methoxyphenyl)-1-phenyl-3-phenylsulfenyl-propan-1-one (3i). White solid; 83-85 ${ }^{\circ} \mathrm{C}$; Rf (10\% AcOEt/hexane) 0.26; IR (KBr, $\left.v \mathrm{~cm}^{-1}\right): 1730 \mathrm{~cm}^{-1} ;{ }^{1} \mathrm{H} \mathrm{NMR}\left(400 \mathrm{MHz}, \mathrm{CDCl}_{3}\right) \delta$ : $3.62\left(2 \mathrm{H}, \mathrm{dd}, \mathrm{J}_{1}=7.5 \mathrm{~Hz}, \mathrm{~J}_{2}=15.2 \mathrm{~Hz}, \mathrm{~J}_{3}=17.2 \mathrm{~Hz}, \mathrm{CH}_{2}\right), 3.76\left(3 \mathrm{H}, \mathrm{s}, \mathrm{OCH}_{3}\right) ; 4,92\left(\mathrm{H}, \mathrm{dd}, \mathrm{J}_{1}=\right.$ $\left.6,2 \mathrm{~Hz}, \mathrm{~J}_{2}=8.1 \mathrm{~Hz}, \mathrm{CH}\right), 6.78(2 \mathrm{H}, \mathrm{m}, \mathrm{J}=8.6 \mathrm{~Hz}$, arom $), 7.1-8.05(12 \mathrm{H}, \mathrm{m}$, arom $) ;{ }^{13} \mathrm{C}$ NMR $(100$ $\left.\mathrm{MHz}, \mathrm{CDCl}_{3}\right) \delta: 197.19,158.77,144.7,136.78,134.48,133.13,129.1,128.63,128.09,127.47$, 119.82, 114.46, 113.86, 55.33, 47.67, 44.86; $\mathrm{MS}(\mathrm{m} / \mathrm{z}): 348\left(\mathrm{M}^{+}\right), 239,238,237,109$.

3-(4-Nitrophenyl)-1-phenyl-3-phenylsulfenyl-propan-1-one (3j). White solid; 84-87 ${ }^{\circ} \mathrm{C}$; Rf (10\% AcOEt/hexane) 0.2; IR (KBr): $1720 \mathrm{~cm}^{-1} ;{ }^{1} \mathrm{H} \mathrm{NMR}\left(400 \mathrm{MHz}, \mathrm{CDCl}_{3}\right) \delta: 3.6\left(2 \mathrm{H}, \mathrm{d}, \mathrm{J}_{1}=\right.$ $\left.7.5 \mathrm{~Hz}, \mathrm{CH}_{2}\right), 4,91\left(\mathrm{H}, \mathrm{t}, \mathrm{J}_{1}=7.5 \mathrm{~Hz}, \mathrm{CH}\right), 7.13-7.5(10 \mathrm{H}, \mathrm{m}, \operatorname{arom}), 7.79\left(2 \mathrm{H}, \mathrm{d}, \mathrm{J}_{1}=7.56 \mathrm{~Hz}\right.$, 
arom), $8\left(2 \mathrm{H}, \mathrm{d}, \mathrm{J}_{1}=7.56 \mathrm{~Hz}\right.$, arom); ${ }^{13} \mathrm{C} \mathrm{NMR}\left(100 \mathrm{MHz}, \mathrm{CDCl}_{3}\right) \delta: 196.11,149.17,146.94$, $136.28,133.68,133.48,132.78,129.13,128.79,128.73,128.36,128.05,123.66,47.90,44.07$; MS (m/z): $363\left(\mathrm{M}^{+}\right), 253,109$.

3-(2-Aminophenylsulfenyl)-1,3-diphenylpropan-1-one (3k). White solid; mp 126-128 ${ }^{\circ} \mathrm{C}$; $\mathrm{Rf}$ (10\% AcOEt/hexane) $0.20 ; v_{\max }(\mathrm{KBr}) 1710 ; 3410 \mathrm{~cm}^{-1} ; \delta_{\mathrm{H}}\left(400 \mathrm{MHz} \mathrm{CDCl}_{3}\right) 3.5\left(\mathrm{H}, \mathrm{CH}_{2}, \mathrm{dd}\right.$, $\left.J_{1}=7.0 \mathrm{~Hz}, J_{2}=17.4 \mathrm{~Hz}\right) ; 3.7\left(\mathrm{H}, \mathrm{CH}_{2}, \mathrm{dd}, J_{1}=7.3 \mathrm{~Hz}, J_{2}=17.4 \mathrm{~Hz}\right) ; 4.3\left(2 \mathrm{H}, \mathrm{NH}_{2}, \mathrm{bs}\right) ; 4,73$ $(\mathrm{H}, \mathrm{CH}, \mathrm{t}, J=7.1 \mathrm{~Hz}) ; 6.53-7.54\left(12 \mathrm{H}\right.$, arom, m) ; $7.9(2 \mathrm{H}$, arom, d, $J=7.2 \mathrm{~Hz}) ; \delta_{\mathrm{C}}(100 \mathrm{MHz}$ $\left.\mathrm{CDCl}_{3}\right) 44.1 ; 47.1 ; 114.9 ; 115.3 ; 115.7 ; 118.0 ; 118.2 ; 127.3 ; 127.6 ; 128.1 ; 128.4 ; 128.6$; $130.6 ; 131.6 ; 133.3 ; 136.8 ; 137.7 ; 141.7 ; 148.7 ; 149.5 ; 197.2 ; \mathrm{MS}(\mathrm{m} / \mathrm{z}): 333\left(\mathrm{M}^{+}\right) ; 207$; 126.

3-(2-Aminophenylsulfenyl)-3-(4-chlorophenyl)-1-phenylpropan-1-one (3l). White solid; mp $78-81{ }^{\circ} \mathrm{C} ; \mathrm{R}_{\mathrm{f}}\left(10 \%\right.$ AcOEt/hexane) $0.33 ; v_{\max }(\mathrm{KBr}) 1700 ; 3470 \mathrm{~cm}^{-1} ; \delta_{\mathrm{H}}\left(400 \mathrm{MHz} \mathrm{CDCl}_{3}\right) 3.4$ $\left(\mathrm{H}, \mathrm{CH}_{2}, \mathrm{dd}, J_{1}=7.1 \mathrm{~Hz}, J_{2}=17.5 \mathrm{~Hz}\right) ; 3.6\left(\mathrm{H}, \mathrm{CH}_{2}, \mathrm{dd}, J_{1}=7.2 \mathrm{~Hz}, J_{2}=17.5 \mathrm{~Hz}\right) ; 4.1(2 \mathrm{H}$, $\left.\mathrm{NH}_{2}, \mathrm{bs}\right) ; 4.9(1 \mathrm{H}, \mathrm{CH}, \mathrm{t}, J=7.1 \mathrm{~Hz}) ; 6.5-7,5(11 \mathrm{H}$, arom, m) ; 7.9 (2H, arom, d, $J=7.2 \mathrm{~Hz})$; $\delta_{\mathrm{C}}\left(100 \mathrm{MHz} \mathrm{CDCl}_{3}\right) 43.4 ; 45.8 ; 115.2 ; 118.3 ; 118.8 ; 122.2 ; 122.5 ; 128.1 ; 128.7 ; 129.1$; $131.1 ; 131.6 ; 133.6 ; 134.1 ; 136.8 ; 137.5 ; 148.6 ; 149.4 ; 196 . \mathrm{MS}(\mathrm{m} / \mathrm{z}) 367\left(\mathrm{M}^{+}\right) ; 242 ; 111$.

3-(2-Aminophenylsulfenyl)-3-(4-methoxyphenyl)-1-phenyl-propan-1-one (3m). White solid; 108-111 $\quad{ }^{\circ} \mathrm{C}$; $\quad$ Rf: $\quad(10 \quad \% \quad$ AcOEt/hexane $) \quad 0.24 ; \quad$ IR $\quad(\mathrm{KBr}, \quad v$ $\left.\mathrm{cm}^{-1}\right): 3420,1730 \mathrm{~cm}^{-1} ;{ }^{1} \mathrm{H}$ NMR $\left(400 \mathrm{MHz}, \mathrm{CDCl}_{3}\right) \delta: 3.52\left(2 \mathrm{H}, \mathrm{dd}, \mathrm{J}_{1}=7.5 \mathrm{~Hz}, \mathrm{~J}_{2}=15.2 \mathrm{~Hz}\right.$, $\left.\mathrm{J}_{3}=17.3 \mathrm{~Hz}, \mathrm{CH}_{2}\right), 3.76\left(3 \mathrm{H}, \mathrm{s}, \mathrm{OCH}_{3}\right), 4.2\left(2 \mathrm{H}, \mathrm{s}, \mathrm{NH}_{2}\right), 4,8\left(\mathrm{H}, \mathrm{dd}, \mathrm{J}_{1}=6,2 \mathrm{~Hz}, \mathrm{~J}_{2}=8.1 \mathrm{~Hz}, \mathrm{CH}\right)$, 6.48-7.76 (11H, m, arom), $7.95\left(2 \mathrm{H}, \mathrm{d}, \mathrm{J}=7.4 \mathrm{~Hz}\right.$, arom); ${ }^{13} \mathrm{C} \mathrm{NMR}\left(100 \mathrm{MHz}, \mathrm{CDCl}_{3}\right) \delta$ : $19736,158.74,149.5,137.69,136.33,135.10,133.25,131.13,130.62,129.71,129.72,128.10$, $127.49,127.19,125.32,123.01,118.07,114.88,114.09,113.77,55.30,46.32,44.32 ; \mathrm{MS}(\mathrm{m} / \mathrm{z})$ : $363\left(\mathrm{M}^{+}\right), 239,238,237,125$.

3-(2-Aminophenylsulfenyl)-3-(4-nitrophenyl)-1-phenylpropan-1-one (3n). White solid; 126$129 \quad{ }^{\circ} \mathrm{C} ; \quad \mathrm{Rf} \quad(10 \quad \% \quad$ AcOEt/hexane $) \quad 0.20 ; \quad \mathrm{IR} \quad(\mathrm{KBr}, \quad v$ $\left.\mathrm{cm}^{-1}\right): 3470,1680 \mathrm{~cm}^{-1} ;{ }^{1} \mathrm{H}$ NMR $\left(400 \mathrm{MHz}, \mathrm{CDCl}_{3}\right) \delta: 3.5\left(\mathrm{H}, \mathrm{dd}, \mathrm{J}_{1}=7.2 \mathrm{~Hz}, \mathrm{~J} 2=17.7 \mathrm{~Hz}\right.$, $\left.\mathrm{CH}_{2}\right), 3.7\left(\mathrm{H}, \mathrm{dd}, \mathrm{J}_{1}=7.1 \mathrm{~Hz}, \mathrm{~J} 2=17.7 \mathrm{~Hz}, \mathrm{CH}_{2}\right), 4.4\left(2 \mathrm{H}, \mathrm{s}, \mathrm{NH}_{2}\right), 4,8(\mathrm{H}, \mathrm{t}, \mathrm{J}=7.1 \mathrm{~Hz}, \mathrm{CH}), 6.4-$ $8.2\left(13 \mathrm{H}, \mathrm{m}\right.$, arom); ${ }^{13} \mathrm{C} \mathrm{NMR}\left(100 \mathrm{MHz}, \mathrm{CDCl}_{3}\right) \delta: 196.35,149.33,147.32,137.52,136.83$, $135.05,133.69$, 131.62, 131.51, 130.39, 128.93, 128.80, 128.52, 128.08, 118.30, 115.31, 115.10, 45.94, 43.55; $\mathrm{MS}(\mathrm{m} / \mathrm{z}): 378\left(\mathrm{M}^{+}\right), 208,123$.

3-Benzylamino-1,3-diphenylpropan-1-one (3o). White solid; mp 75-76 ${ }^{\circ} \mathrm{C} ; \quad \mathrm{R}_{\mathrm{f}} \quad(10 \%$ AcOEt/hexane) $0.41 ; v_{\max }(\mathrm{KBr}) 1690 ; 3030 ; 3380 \mathrm{~cm}^{-1} ; \delta_{\mathrm{H}}\left(400 \mathrm{MHz} \mathrm{CDCl}_{3}\right) 2.0(1 \mathrm{H}, \mathrm{NH}$, bs) ; $3.3\left(\mathrm{H}, \mathrm{CH}_{2}\right.$, dd, $\left.J_{1}=4.8 \mathrm{~Hz}, J_{2}=17.1 \mathrm{~Hz}\right) ; 3.4\left(\mathrm{H}, \mathrm{CH}_{2}, \mathrm{dd}, J_{1}=8 \mathrm{~Hz}, J_{2}=17.1 \mathrm{~Hz}\right) ; 3.6$ $\left(2 \mathrm{H}, \mathrm{CH}_{2}, \mathrm{~m}\right) ; 4.3\left(1 \mathrm{H}, \mathrm{CH}, \mathrm{dd}, J_{1}=4.8 \mathrm{~Hz}, \mathrm{~J}_{2}=8 \mathrm{~Hz}\right) ; 7.2-7.6(13 \mathrm{H}$, arom, m) ; 7.9 (2H, arom, $\mathrm{d}, J=7.5 \mathrm{~Hz}) ; \delta_{\mathrm{C}}\left(100 \mathrm{MHz} \mathrm{CDCl}_{3}\right) 46.5 ; 47.3 ; 51.6 ; 58.5 ; 122.1 ; 126.8 ; 127.1 ; 127.3$; $128.1 ; 128.3 ; 128.6 ; 128.7 ; 129.0 ; 130.5 ; 132.8 ; 133.2 ; 140.4 ; 143.2 ; 144.8 ; 198.9$; MS $(\mathrm{m} / \mathrm{z}): 195\left(\mathrm{M}-\mathrm{C}_{6} \mathrm{H}_{5} \mathrm{COCH}_{3}\right), 165,152,120\left(\mathrm{M}-\mathrm{C}_{6} \mathrm{H}_{5} \mathrm{CH}=\mathrm{NCH}_{2} \mathrm{C}_{6} \mathrm{H}_{5}\right), 117 ; 105,91,77,65$, 51 . 
3-Benzylamino-3-(4-chlorophenyl)-1-phenylpropan-1-one (3p). White solid; mp 98-100 ${ }^{\circ} \mathrm{C}$; $\mathrm{R}_{\mathrm{f}} \quad(10 \% \quad$ AcOEt/hexane $) \quad 0.42 ; \quad \mathrm{U}_{\max } \quad(\mathrm{KBr}) 1690 ; \quad 3030$; $3380 \mathrm{~cm}^{-1} ; \delta_{\mathrm{H}}\left(400 \mathrm{MHz} \mathrm{CDCl}_{3}\right) 2.2(1 \mathrm{H}, \mathrm{NH}, \mathrm{bs}) ; 3.2\left(\mathrm{H}, \mathrm{CH}_{2}, \mathrm{dd}, J_{1}=4.8 \mathrm{~Hz}, J_{2}=17.1 \mathrm{~Hz}\right)$; $3.3\left(\mathrm{H}, \mathrm{CH}_{2}, \mathrm{dd}, J_{1}=8 \mathrm{~Hz}, J_{2}=17.1 \mathrm{~Hz}\right) ; 3.6\left(2 \mathrm{H}, \mathrm{CH}_{2}, \mathrm{~m}\right) ; 4.3\left(1 \mathrm{H}, \mathrm{CH}, \mathrm{dd}, J_{1}=4.8 \mathrm{~Hz}, J_{2}=8\right.$ $\mathrm{Hz}) ; 7.2-7.6\left(12 \mathrm{H}\right.$, arom, m) ; $7.9(2 \mathrm{H}$, arom, d, $J=7.5 \mathrm{~Hz}) ; \delta_{\mathrm{C}}\left(100 \mathrm{MHz} \mathrm{CDCl}_{3}\right) 47.8 ; 51.6$; $52.1 ; 126.8 ; 128.1 ; 128.3 ; 128.4 ; 128.6 ; 128.7 ; 129.5 ; 132.9 ; 135.3 ; 137.2 ; 137.4 ; 197.6$; MS (m/z): $229\left(\mathrm{M}-\mathrm{C}_{6} \mathrm{H}_{5} \mathrm{COCH}_{3}\right), 194,151,125,120\left(\mathrm{M}-p-\mathrm{ClC}_{6} \mathrm{H}_{5} \mathrm{CH}=\mathrm{NCH}_{2} \mathrm{C}_{6} \mathrm{H}_{5}\right), 105$, $91,77,65,51$.

3-Benzylamino-3-(4-methoxyphenyl)-1-phenylpropan-1-one (3q). White solid; mp 80-81 ${ }^{\circ} \mathrm{C}$; $\mathrm{R}_{\mathrm{f}}\left(10 \%\right.$ AcOEt/hexane) $0.37 ; \mathrm{v}_{\max }(\mathrm{KBr}) 1688 ; 3030 ; 3370 \mathrm{~cm}^{-1} ; \delta_{\mathrm{H}}\left(400 \mathrm{MHz} \mathrm{CDCl}_{3}\right) 2.2$ $(1 \mathrm{H}, \mathrm{NH}, \mathrm{bs}) ; 3.3\left(\mathrm{H}, \mathrm{CH}_{2}, \mathrm{dd}, J_{1}=4.8 \mathrm{~Hz}, J_{2}=17.1 \mathrm{~Hz}\right) ; 3.4\left(\mathrm{H}, \mathrm{CH}_{2}, \mathrm{dd}, J_{1}=8 \mathrm{~Hz}, J_{2}=17.1\right.$ $\mathrm{Hz}) ; 3.6\left(2 \mathrm{H}, \mathrm{CH}_{2}, \mathrm{~m}\right) ; 3.8\left(3 \mathrm{H}, \mathrm{OCH}_{3}, \mathrm{~s}\right) ; 4,3\left(1 \mathrm{H}, \mathrm{CH}, \mathrm{dd}, J_{1}=4.8 \mathrm{~Hz}, J_{2}=8 \mathrm{~Hz}\right) ; 7.2-7,6$ $\left(12 \mathrm{H}\right.$, arom, m) $7.9(2 \mathrm{H}$, arom, d, $J=7.5 \mathrm{~Hz}) ; \delta_{\mathrm{C}}\left(100 \mathrm{MHz}_{\mathrm{CDCl}}\right) 47.8 ; 51.6 ; 52.1 ; 56.0$; $113.9 ; 126.8 ; 128.1 ; 128.3 ; 128.4 ; 128.6 ; 129.1 ; 129.5 ; 132.9 ; 137.2 ; 137.4 ; 197.6$; MS $(\mathrm{m} / \mathrm{z}): 225\left(\mathrm{M}-\mathrm{C}_{6} \mathrm{H}_{5} \mathrm{COCH}_{3}\right), 210,194,147,134,120\left(\mathrm{M}-p-\mathrm{MeOC}_{6} \mathrm{H}_{4} \mathrm{CH}=\mathrm{NCH}_{2} \mathrm{C}_{6} \mathrm{H}_{5}\right), 117$, $105,91,77,65,51$.

1,3-Diphenyl-3-phenylaminopropan-1-one (3r). White solid; $167-169 \quad{ }^{\circ} \mathrm{C} ; \quad \mathrm{R}_{\mathrm{f}} \quad(10 \%$ AcOEt/hexane) $0.24 ; v_{\max }(\mathrm{KBr}) 1690 ; 3030 ; 3400 \mathrm{~cm}^{-1} ; \delta_{\mathrm{H}}\left(400 \mathrm{MHz} \mathrm{CDCl}_{3}\right) 3.4\left(\mathrm{H}, \mathrm{CH}_{2}, \mathrm{dd}\right.$, $\left.J_{1}=7.6 \mathrm{~Hz}, J_{2}=16 \mathrm{~Hz}\right) ; 3.5\left(\mathrm{H}, \mathrm{CH}_{2}, \mathrm{dd}, J_{1}=5.2 \mathrm{~Hz}, J_{2}=16 \mathrm{~Hz}\right) ; 4,5(1 \mathrm{H}, \mathrm{NH}, \mathrm{Bs}) ; 4.99(\mathrm{H}$, $\mathrm{CH}, \mathrm{t}, J=5.2 \mathrm{~Hz}) ; 6.55-7.9\left(13 \mathrm{H}\right.$, arom, m) ; $7.9(2 \mathrm{H}$, arom, d, $J=7.5 \mathrm{~Hz}) ; \delta_{\mathrm{C}}(100 \mathrm{MHz}$ $\left.\mathrm{CDCl}_{3}\right) 38.8 ; 41.2 ; 78.9 ; 122.3 ; 122.9 ; 123.2 ; 124.5 ; 128.0 ; 128.8 ; 130.0 ; 133.8 ; 134.4$; $136.0 ; 148.5 ; 196.4 ; \mathrm{MS}(\mathrm{m} / \mathrm{z}): 181\left(\mathrm{M}-\mathrm{C}_{6} \mathrm{H}_{5} \mathrm{COCH}_{3}\right), 152,120\left(\mathrm{M}-\mathrm{C}_{6} \mathrm{H}_{5} \mathrm{CH}=\mathrm{NC}_{6} \mathrm{H}_{5}\right), 105$, $104,77,51$.

3-(4-Chlorophenyl)-1-phenyl-3-phenylaminopropan-1-one (3s). White solid, 110-111 ${ }^{\circ} \mathrm{C} ; \mathrm{R}_{\mathrm{f}}$ (10\% AcOEt/hexane) $0.25 ; v_{\max }(\mathrm{KBr}) 1690 ; 3030 ; 3400 \mathrm{~cm}^{-1} ; \delta_{\mathrm{H}}\left(400 \mathrm{MHz} \mathrm{CDCl}_{3}\right) 3.3(\mathrm{H}$, $\left.\mathrm{CH}_{2}, \mathrm{dd}, J_{1}=7 \mathrm{~Hz}, J_{2}=16.2 \mathrm{~Hz}\right) ; 3.4\left(\mathrm{H}, \mathrm{CH}_{2}, \mathrm{dd}, J_{1}=5.5 \mathrm{~Hz}, J_{2}=16.2 \mathrm{~Hz}\right) ; 4.5(1 \mathrm{H}, \mathrm{NH}, \mathrm{bs})$; $4.9(1 \mathrm{H}, \mathrm{CH}, \mathrm{t}, J=6.5 \mathrm{~Hz}) ; 6.5(2 \mathrm{H}$, arom, d, $J=7.7 \mathrm{~Hz}) ; 6.6(\mathrm{H}$, arom, t, $J=7.2 \mathrm{~Hz}) ; 7.1(2 \mathrm{H}$, arom, t, $J=7.7 \mathrm{~Hz}) ; 7.2-7.4(6 \mathrm{H}$, arom, $\mathrm{m}) ; 7.5(1 \mathrm{H}$, arom, t, $J=7.7 \mathrm{~Hz}) ; 7.9(2 \mathrm{H}$, arom, d, $J=$ $7.2 \mathrm{~Hz}) ; \delta_{\mathrm{C}}\left(100 \mathrm{MHz} \mathrm{CDCl}_{3}\right) 46.1 ; 54.1 ; 113.8 ; 118.0 ; 127.8 ; 128.1 ; 128.7 ; 128.9 ; 129.1$; $132.9 ; 133.5 ; 136.5 ; 141.5 ; 146.7 ; 197.8 ; \mathrm{MS}(\mathrm{m} / \mathrm{z}): 215\left(\mathrm{M}-\mathrm{C}_{6} \mathrm{H}_{5} \mathrm{COCH}_{3}\right), 180,152,120$ $\left(\mathrm{M}-p-\mathrm{ClC}_{6} \mathrm{H}_{5} \mathrm{CH}=\mathrm{NC}_{6} \mathrm{H}_{5}\right), 112,105,89,77,51$.

3-(4-Methoxyphenyl)-1-phenyl-3-phénylaminopropan-1-one (3t). White solid, 135-137 ${ }^{\circ} \mathrm{C}$; $\mathrm{R}_{\mathrm{f}}\left(10 \%\right.$ AcOEt/hexane) $0.12 ; v_{\max }(\mathrm{KBr}) 1690 ; 3030 ; 3400 \mathrm{~cm}^{-1} ; \delta_{\mathrm{H}}\left(400 \mathrm{MHz} \mathrm{CDCl}_{3}\right) 3.3(\mathrm{H}$, $\left.\mathrm{CH}_{2}, \mathrm{dd}, J_{1}=7.2 \mathrm{~Hz}, J_{2}=16 \mathrm{~Hz}\right) ; 3.4\left(\mathrm{H}, \mathrm{CH}_{2}, \mathrm{dd}, J_{1}=5.6 \mathrm{~Hz}, J_{2}=16 \mathrm{~Hz}\right) ; 3.76\left(3 \mathrm{H}, \mathrm{OCH}_{3}\right.$, s) ; $4.5(1 \mathrm{H}, \mathrm{NH}, \mathrm{bs}) ; 4.98(1 \mathrm{H}, \mathrm{CH}, \mathrm{t}, J=6.5 \mathrm{~Hz}) ; 6.5(2 \mathrm{H}$, arom, d, $J=8.2 \mathrm{~Hz}) ; 6.6(\mathrm{H}$, arom, $\mathrm{t}, J=7.2 \mathrm{~Hz}) ; 6.8(2 \mathrm{H}$, arom, d, $J=8.5 \mathrm{~Hz}) ; 7.1(2 \mathrm{H}$, arom, t, $J=7.5 \mathrm{~Hz}) ; 7.3(2 \mathrm{H}$, arom, d, $J=$ $8.7 \mathrm{~Hz}) ; 7.4(2 \mathrm{H}$, arom, t, $J=7.7 \mathrm{~Hz}) ; 7.5(\mathrm{H}$, arom, t, $J=7.2 \mathrm{~Hz}) ; 7.9(2 \mathrm{H}$, arom, d, $J=7.2$ $\mathrm{Hz}) ; \delta_{\mathrm{C}}\left(100 \mathrm{MHz} \mathrm{CDCl}_{3}\right) 46.3 ; 54.2 ; 55.2 ; 113.8 ; 114.1 ; 117.7 ; 127.4 ; 128.2 ; 128.6$; $129.1 ; 133.3 ; 134.9 ; 136.7 ; 147.0 ; 158.8 ; 198.4 ; \mathrm{MS}(\mathrm{m} / \mathrm{z}): 211\left(\mathrm{M}-\mathrm{C}_{6} \mathrm{H}_{5} \mathrm{COCH}_{3}\right), 195$, $180,167,120\left(\mathrm{M}-p-\mathrm{MeOC}_{6} \mathrm{H}_{5} \mathrm{CH}=\mathrm{NC}_{6} \mathrm{H}_{5}\right), 105,104,77,51$. 


\section{References and Notes}

1. (a) Sasai, T.; Arai, T.; Satow, Y.; Houk, K. N.; Shibasaki, M. J. Am. Chem. Soc. 1995, 117, 6194. (b) Fernández, I.; Araújo, C. S.; Romero, J.; Alcudia, F.; Khiar, N. Tetrahedron. 2000, 56, 3749. (c) Lee, P. H.; Ahn, H.; Lee, K.; Sung, S.; Kim S. Tetrahedron Lett. 2001, 42, 37. (d) Shaikh, N. S.; Deshpande, V. H.; Bedekar, A. V. Tetrahedron. 2001, 57, 9045. (e) Cheng, S.; Cromer D. D. Tetrahedron Lett. 2002, 43, 1179.

2. Zahouily, M.; Abrouki, Y.; Rayadh, A.; Sebti, S.; Dhimane, H.; David, M. Tetrahedron Lett. 2003, 44, 2463.

3. Zahouily, M. Abrouki, Y. Rayadh, A. Tetrahedron Lett. 2002, 43, 7729.

4. Sharma, U.; Bora, U.; Boruah, R. C.; Sandhu, J. S. Tetrahedron Lett. 2002, 43, 143.

5. Choudary, B. M.; Kantam, M. L.; Reddy, C. R. V.; Rao, K. K.; Figueras, F. J. Mol. Catal. A: Chemical. 1999, 146, 279.

6. (a) Zahouily, M.; Bahlaouan, O.; Bahlaouan, B.; Rayadh, A.; Sebti, S. Arkivoc. 2005, 13, 150. (b) Zahouily, M.; Charki, H.; Abrouki, Y.; Mounir, B.; Bahlaouan, B.; Rayadh, A.; Sebti, S. Lett. in Org. Chem. 2005, 2, 136. (c) Zahouily, M.; Bahlaouan, B.; Aadil, M.; Rayadh, A.; Sebti, S. Org. Process Research \& Development. 2004, 8, 275.

7. (a) Abrouki, Y.; Zahouily, M.; Rayadh, A.; Bahlaouan, B.; Sebti, S. Tetrahedron Lett. 2002, 43, 8951. (b) Zahouily, M.; Mezdar, A.; Elmakssoudi, A.; Mounir, B.; Rayadh, R.; Sebti, S.; Lazrek, H. B. Arkivoc 2006, (ii), 42.

8. Bennazha, J.; Zahouily, M.; Boukhari, A.; Holt, E. M. J. Mol. Catal. A: Chemical. 2003, 202, 247.

9. Li, J.-T.; Chen, G. -F.; Xu, W.-Z.; Li, T.-S. Ultrason Sonochem. 2003, 10, 115.

10. Sharma, U.; Bora, U.; Boruh, R. C.; Sandhu, J. S. Tetrahedron Lett. 2002, 43, 143.

11. Zahouily, M.; Bahlaouan, B.; Solhy, A.; Ouammou, M.; Sebti, S. React. Kinet. Catal. Lett. 2003, 78, 129

12. Ouammou, M.; Benhassaine, A. ; Nadiri, A. Adv. Mat. Research. 1994, 1-2, 621.

13. Ouammou, M.; Clerc, L.; Benhassaine, A. ; Nadiri, A. Adv. Mat. Research. 1994, 1-2, 243.

14. Roudier, J. F; Foucaud, A. Synth. Commun. 1984, 159.

15. Garcia-Raso, A.; Garcia-Raso, J.; Campaner, B.; Mestres, R.; Sinisterra, J. V. Synthesis 1982, 12, 1037.

16. Watanabe, K. I.; Miyazu, K. I.; Irie, K. Bull. Chem. Soc. Jpn. 1982, 55, 3212.

17. Li, J. T. ; Chen, G. F. ; Xu, W. Z. ; Li, T. S. Ultra. Sonochemistry 2003, 10, 115. 